\title{
Traduire
}

Revue française de la traduction

$231 \mid 2014$

A table !

\section{Un thé indien avec Dominique Vitalyos}

Propos recueillis par Lakshmi Ramakrishnan Iyer

Dominique Vitalyos et Lakshmi Ramakrishnan lyer

\section{OpenEdition}

Journals

Édition électronique

URL : http://journals.openedition.org/traduire/659

DOI : $10.4000 /$ traduire. 659

ISSN : 2272-9992

Éditeur

Société française des traducteurs

Édition imprimée

Date de publication : 1 décembre 2014

Pagination : 43-51

ISSN : 0395-773X

Référence électronique

Dominique Vitalyos et Lakshmi Ramakrishnan lyer, « Un thé indien avec Dominique Vitalyos », Traduire [En ligne], 231 | 2014, mis en ligne le 01 décembre 2016, consulté le 20 avril 2019. URL : http:// journals.openedition.org/traduire/659; DOI : 10.4000/traduire.659 


\title{
Un thé indien avec Dominique Vitalyos
}

\author{
Propos recueillis par Lakshmi Ramakrishnan lyer
}

Dans cet entretien passionné, Dominique Vitalyos, traductrice de littérature indienne, nous dévoile son parcours pour le moins insolite. Son apprentissage laborieux du malayalam, l'une des 22 langues officielles de l'Inde; ses débuts dans la traduction littéraire, qui font croire à l'existence du destin ; sa traduction d'un recueil de nouvelles gastronomiques qui met l'eau à la bouche...

Vous êtes traductrice littéraire du malayalam et de l'anglais vers le français pour le domaine indien. Le malayalam fait partie, comme les trois autres langues officielles du sud de l'Inde, des langues dravidiennes dont la structure ne présente aucune parenté avec les langues indo-européennes et qui ont, de ce fait, la réputation d'être difficiles à apprendre. Quel parcours avez-vous suivi ?

Au début des années 1980, curieuse de comprendre la façon dont les Malayali(1) voyaient le monde et envisageaient la vie, j'ai décidé de plonger dans l'étude du malayalam. J'avais appris l'écriture ainsi que quelques rudiments de vocabulaire auprès d'un Malayali qui habitait Paris. J'avais également suivi les cours de première année de tamoul, la langue sœur du malayalam, à Langues 0'. En 1985, j'ai postulé à une bourse au titre des échanges culturels indo-français (qui existaient alors) pour apprendre le malayalam sur place et je l'ai obtenue. J'ai aussitôt quitté mon travail de bibliothécaire à l'Institut Pasteur de Paris sans la moindre appréhension pour ce qui m'attendait.

Au Kerala, on m'a inscrite d'office dans une école " internationale de linguistique dravidienne ". Si dans la phrase ci-dessus j'ai laissé "école " hors des guillemets, c'est qu'il s'agissait plutôt d'une sorte d'institut de recherche dans lequel aucun des chercheurs n'avait la moindre idée de la façon d'enseigner le malayalam à un non-Dravidien. II n'existait aucune méthode, aucun précédent institutionnel. On détachait auprès de moi un enseignant du secondaire pour

(1) Les habitants du Kerala, État du sud-ouest de l'Inde. 
quelques mois, puis un autre, sans grand succès. J'avais besoin de comprendre, et personne ne m'expliquait quoi que ce soit. Pour moi, apprendre une langue, c'était (en deux mots) en aborder les mécanismes, les éléments constitutifs et leurs combinatoires pour créer mon propre discours. Répéter, appliquer, passe encore, mais lorsqu'une fois sur trois on tombe sur une exception à la soi-disant règle sans savoir pourquoi, c'est désespérant! (Par exemple, si vous apprenez à dire en malayalam " je ne viens pas ", ne croyez pas pour autant avoir appris à dire " je " ou " ne pas " : nombre de " nos " cas sujets se vivent au " datif " en malayalam, et la façon d'exprimer la négation varie en fonction du verbe... et du mode...)

Dehors, je faisais semblant de ne pas connaître l'anglais pour que l'on s'adresse à moi systématiquement en malayalam. Le peu que je parlais, je le faisais en imitant la prononciation et l'accent, ce qui curieusement m'entraînait sur de bonnes pistes et, sans doute par un effet de mémoire phonétique subconsciente de ce que j'avais déjà entendu, m'empêchait de commettre certaines erreurs.

J'ai commencé très tôt à essayer de lire des textes à mon rythme. Car si vous ne répondiez pas du tac au tac, les professeurs vous fournissaient la réponse illico, entravant la compréhension qui progresse avec le cheminement personnel de l'intellect, démolissant tout l'intérêt et le plaisir de la réflexion.

J'ai consulté des grammaires, mais elles ne m'ont pas beaucoup aidée. Dans la plupart d'entre elles, les auteurs tentent de tout faire coïncider avec des catégories souvent préétablies à partir du... sanskrit (langue qui n'a rien de dravidien) ! Définissant ainsi des règles beaucoup trop bancales pour prétendre s'exprimer en malayalam quand on les applique systématiquement.

À l'institut, on a enfin trouvé un moyen pour que j'avance : on m'a confiée à un des chercheurs qui parlait bien anglais et s'intéressait à l'actualité, et nous avons lu le journal malayalam ensemble. Je l'arrêtais chaque fois que j'avais besoin d'une explication. S'il ne savait pas répondre (il n'était pas grammairien, moi non plus), je suggérais des hypothèses, et nous finissions, de proche en proche, par apporter une réponse à mon interrogation. J'ai donc appris à poser des questions de plus en plus ciblées et subtiles. De ce point de vue, le service que m'a rendu cette école est inestimable. Elle m'a permis d'envisager un nouvel abord grammatical du malayalam à l'usage des francophones (que j'ai commencé à tester avec succès) dans un manuel dont je reprendrai bientôt, j'espère, la rédaction...

\section{Envisagiez-vous de faire de la traduction votre métier, à cette époque ? Comment est- ce arrivé ?}

Pas du tout! Je ne me prédestinais pas plus à ce métier qu'à un autre lorsque j'ai quitté la France. Je souhaitais vivre au Kerala, y étudier le malayalam et le kathakali(2) par pur plaisir. À bien regarder, cependant, de nombreux ingrédients concernant l'Autre, sa langue et la mienne,

(2) Le théâtre dansé du Kerala, qui met en scène les dieux, héros et démons de la mythologie hindoue. 
étaient déjà en place : j'aimais écrire, j'avais suivi des études d'ethnologie, d'anglais, d'indonésien et de civilisation asiatique ; au lycée, déjà, je jonglais avec les langues étrangères, et une enseignante de lettres, que je ne remercierai jamais assez, m'avait proposé de me présenter au concours général. En bonne adolescente rebelle, j'avais refusé, mais elle m'avait donné par ce signe, mine de rien, confiance en mes capacités à m'exprimer dans ma langue maternelle.

Au Kerala, ma passion pour le kathakali s'est concentrée sur Nala Charitam, un des joyaux du répertoire, contribuant de façon décisive à cette évolution vers le métier de traductrice. Nala Charitam, l'histoire merveilleuse d'un amour entre un jeune roi (Nala) et son épouse (Damayanti), mis à rude épreuve par un esprit malin, est un conte inséré dans la grande épopée panindienne du Mahabharata. La pièce qu'en a tirée Unnâyi Vâriyar pour le kathakali est l'une des œuvres les plus admirables de la littérature malayalam. Servie par une conjonction d'interprètes - acteurs et musiciens - de génie, elle m'a emportée dans un tourbillon de charme au sens le plus fort. Un beau jour, je me suis retrouvée un crayon à la main, traduisant entre les lignes du texte original, poussée - je l'ai compris plus tard - par le besoin d'intégrer ma langue maternelle (que je ne parlais plus depuis cinq ans) à mon identité actualisée et dans mon univers d'élection.

C'était une expérience d'une richesse inouïe. Car au mot malayalam se superposaient devant moi le geste par lequel " parle " l'acteur, l'expression de son visage, sa façon d'incarner le personnage, le mode musical des chanteurs, leur voix, leur timbre, autrement dit tout ce qui constitue le rasa, la saveur esthétique dont le partage est le but du théâtre. J'avais l'impression de traduire en relief !

De retour en France, j'ai tenté de trouver un éditeur à ma traduction - Jours d'amour et d'épreuve, l'histoire du roi Nala - sans vraiment y croire, comme on jette une bouteille à la mer. Quand Jacques Dars (Gallimard) a choisi d'en faire le dernier volume de la petite collection Connaissance de l'Orient, en m'autorisant des photos et en me laissant décider de tout, titre et quatrième de couverture inclus, j'ai failli croire aux miracles. J'ai su en tout cas dès ce jourlà qu'il existait des directeurs de collection formidables !

Par ailleurs, Sudhir Kakar, psychanalyste indien de renommée mondiale, m'avait confié la traduction (de l'anglais, cette fois) pour les éditions du Seuil, de Chamans, mystiques et médecins, un de ses ouvrages les plus passionnants sur les différentes manières d'aborder, en Inde traditionnelle, la thérapeutique de ce que nous appelons les troubles mentaux.

Deux traductions - une dans chacune des langues que je partageais avec l'Inde -, deux grands textes, deux grands auteurs, deux grands éditeurs en référence... je me suis rendue à l'évidence. Outre le plaisir que je prenais au travail de traduction proprement dit et à l'écriture en général, en faire mon métier était le meilleur moyen pour moi de gagner ma vie sans trop la perdre et de continuer à fréquenter assidument l'Inde qui m'était devenue profondément familière. 
C'est alors que vous décidez de vous transformer en " entremetteuse ", de proposer aux éditeurs français des livres d'auteurs indiens peu connus et de vous offrir à les traduire.

Oui. Quelques auteurs indiens, vivant tous à l'étranger (Anita Desai, Vikram Seth, Amitav Ghosh, Naipaul), avaient été publiés en France, mais tous les écrivains vivant sur le sol indien étaient parfaitement méconnus (à l'exception de Tagore, bien entendu, et de R.K. Narayan). J'ai décidé d'explorer ce vaste continent, et il y avait de quoi faire ! Bien sûr, il s'agissait surtout d'écrivains anglophones. Je garde un souvenir émerveillé du découvreur de talents infatigable qu'était Ravi Dayal, éditeur à Delhi, chez qui j'ai trouvé plus d'un écrivain aujourd'hui publié en français. Son catalogue était une véritable malle aux trésors. Les textes écrits en malayalam convainquaient rarement les éditeurs, car à cette époque leurs traductions en anglais étaient rares et souvent très mauvaises, et je n'avais pas les moyens de les traduire en français avant de les proposer!

Quelles sont les difficultés spécifiques liées à la traduction du malayalam en français ? Pourriez-vous nous donner des exemples de mots ou de tournures en malayalam qui vous ont donné du fil à retordre et nous expliquer comment vous les avez transposées en français ?

Ce qui est passionnant avec le malayalam, c'est que pratiquement rien n'est traduisible par équivalences ! À commencer par la syntaxe, son verbe en fin de phrase et ses agglutinations impossibles à respecter. Le rendu du sens, de la couleur et du rythme se fait alors par microéquilibrages dans des cadres d'intervention qui dépassent souvent la phrase. L'intraduisible est permanent et pourtant, une histoire de Basheer racontée en français peut faire passer bien des aspects spécifiques de l'original. Ce qui aide le mieux, c'est d'entendre l'histoire racontée en malayalam dans sa tête. Un jour, j'ai lu en malayalam devant un public de traducteurs un extrait de livre (Les Légendes de Khasak, de 0.V. Vijayan). Ils étaient sidérés par le simple relief de l'énoncé, et quand je leur ai lu ma traduction, ils ont eu l'impression d'avoir accédé à quelque chose d'ordre sémantique dès l'écoute du texte en malayalam. Le bonheur !

La difficulté la plus grande est assurément celle de l'expression du temps - cela commence, bien entendu, par sa conception - et des modes verbaux. Ce chapitre est beaucoup trop complexe pour l'aborder en quelques mots. Je dirai simplement ici qu'en fait de temps, le malayalam ne partage avec les langues indo-européennes qu'une certaine forme de passé, et que tout le reste s'articule selon des modes. La restitution de ces nuances en français est un des plus grands défis et des plus grands plaisirs de la traduction!

En ce qui concerne l'aspect lexical, la difficulté que l'on rencontre au début tient souvent au décalage du spectre sémantique de mots "équivalents " l'un par rapport à l'autre. La surface de recouvrement de "love " et " amour " n'est certes pas absolue (p. ex "love ", en position vocative, peut désigner une personne ; ce n'est pas le cas pour " amour ", à moins de lui 
adjoindre un possessif qui change tout), mais le décalage est infiniment plus mince qu'entre " sneham " et " amour ". "Sneham ", le mot malayalam courant (adopté du sanskrit) est à l'origine une qualité physique de fluidité, voire de viscosité, dont l'aspect érotique est totalement occulté au profit de la plus convenable "fluidité " du lien social, elle-même sous-jacente. Rien de tout cela dans " amour ", qui lui nous embarque sans contrainte jusqu'à l'érotique alors que (pour mieux cacher son origine ?) " sneham " ne s'y aventure pas et laisse la place à " premam ".

Je n'ai pas gardé en mémoire les difficultés rencontrées pour traduire un mot ou un autre, à l'exception de l'exemple intéressant et amusant de "kuzhi-âna ", dans Grand-Père avait un éléphant, de V.M. Basheer. L'histoire met en scène, entre autres personnages, une jeune fille à marier et sa mère, très fière de pouvoir lui dire "Ton grand-père avait un éléphant, un grand mâle à défenses ". (Éléphant se dit "âna " en malayalam). À la fin, elle se trouve en butte aux moqueries de petits garnements qui lui crient en l'entendant prononcer sa phrase fétiche : "c'était un kuzhi-âna!" Le mot désigne un petit insecte qui se creuse (kuzhi) un petit nid dans le sol après la pluie, comparé à l'éléphant (âna) pour le semblant de trompe dont il est pourvu.

On s'émerveille en découvrant que le français propose lui aussi un rapprochement avec un grand et noble animal : la petite créature s'appelle (j'ignore pourquoi) un fourmilion. Ce serait une aubaine, si toute l'ironie de la plaisanterie malayalam n'était perdue. L'élément "éléphant " était indispensable pour répondre à la mère ! II a donc fallu inventer, et j'ai opté pour " blattéléphant "qui passe très bien phonétiquement et qui évoque de loin bla-bla et "cause toujours ". De plus, " blatte " a un côté dépréciatif que " puce ", par exemple, n'a pas.

La littérature indienne en anglais est aujourd'hui largement traduite en français ; des éditeurs comme Philippe Picquier ou Albin Michel ont même ajouté ce domaine à leur catalogue. Quant aux manuscrits, ils passent à présent par les mains des agents littéraires. En revanche, vous continuez à proposer des romans en malayalam. Vous avez l'avantage, assez insolite, de pouvoir traduire directement du malayalam en français au lieu de passer par la traduction anglaise. Pensez-vous que ce fait améliore sensiblement la traduction?

Les traductions dites "relais ", effectuées à partir d'une traduction de l'original en anglais, sont à bannir. Si éloignées soient-elles, deux langues telles que le malayalam et le français partagent des liens spécifiques différents de ceux qui sont partagés par le malayalam et l'anglais. Je donne souvent l'exemple de " nân ninne chappâtti âkkum ": en français, " je vais t'aplatir comme une galette ", une merveille d'équivalence (sens et tonalité) que l'anglais ne propose pas, si bien que dans le cas d'une traduction-relais, vous devrez traduire quelque chose comme "l'll beat you into a pulp" par " je vais te réduire en bouillie ", sans savoir ce que vous ratez. Plus généralement, les fils à tisser pour tenter de reproduire le contexte atmosphérique sont 
d'une subtilité qui ne résiste pas à un deuxième passage. À moins que la version anglaise soit l'œuvre d'un génie... ou de l'auteur (qui ne fait pas forcément aussi bien que dans l'original mais qui, au moins, l'aura voulu !).

Philippe Picquier a publié en 1999 votre traduction d'un livre intitulé La colère des aubergines(3), de l'auteure indienne Bulbul Sharma. C'est un recueil de 12 nouvelles drôles, espiègles et finement écrites avec, pour fil rouge, la cuisine. Et, à travers elle, un aperçu de la société indienne, notamment de la vie des femmes en Inde. À la fin de chaque nouvelle, une recette : celle d'un plat dégusté par les personnages dont on vient de suivre les péripéties.

C'est vous qui avez découvert et proposé le livre à l'éditeur. Racontez-nous comment ça s'est passé. Avez-vous rencontré l'auteure?

J'ai contacté les éditions Kali for Women pour connaître leur catalogue et lu The Anger of Aubergines, dont j'ai trouvé très instructif l'abord de la société indienne à travers l'importance qu'elle accorde à la nourriture, et très distrayants le ton et la forme. J'étais presque sûre qu'il plairait au lectorat français et je l'ai aussitôt proposé à Philippe Picquier. J'ai contacté l'autrice (NB : Je suis allergique à la forme " auteur-e ", j'utilise donc " autrice "le féminin naturel d'" auteur ", ici comme ailleurs) un peu plus tard, notamment pour lui demander une recette à base d'aubergines, car il n'en figurait pas dans l'ouvrage, en dépit de son titre et de la nouvelle éponyme. Le français a donc acquis un petit avantage par rapport à l'original... Je dois ajouter que c'est le livre qui m'a rapporté le plus de droits d'auteur, il continue à se vendre chaque année à peu près au même rythme!

Le livre est truffé de noms de plats indiens qui nécessitent une explication. Ces mots en italique apparaissent parfois cinq ou six fois sur une seule page. N'aviez-vous pas la crainte de rebuter le lecteur français avec tous ces mots étrangers en italique? Comment avez-vous traité le problème?

Parfois, je les explicite immédiatement, quand cela peut être fait avec légèreté. Et j'ai ajouté un glossaire à la fin du livre. Certains traducteurs et éditeurs sont contre, mais personnellement j'ai du mal à comprendre pourquoi, puisque le lecteur est évidemment libre de le consulter ou non.

(3) Bulbul Sharma, 1997, The Anger of Aubergines, New Delhi, Kali for Women. 
Connaissiez-vous déjà la plupart de ces plats du fait de vos longs et fréquents séjours en Inde ? Comment vous êtes-vous documentée, notamment pour le glossaire?

Je n'en connaissais que quelques-uns, car je suis plus familière des dosa(4), iddli(5) et autres plats savoureux de l'Inde du Sud. Je cuisine peu, mais chaque année j'invite des amis autour d'un biryani(6) qui me prend une bonne demi-journée de préparation dans les règles de l'art. II faut que j'essaie un jour la recette de Bulbul, très différente. Pour établir le glossaire, je l'ai questionnée sans scrupules!

Les liens de parenté sont très explicites en Inde : il y a un nom spécifique pour chaque membre de la famille. Ainsi la sœur cadette de votre père ne s'appellera pas de la même façon que la sœur cadette de votre mère, alors qu'en français le mot " tante "s'applique aux deux. Comment avez-vous traité le problème des bua et autres masi?

En général, je m'arrange pour signaler le plus tôt possible dans la narration de quel(le) oncle ou tante il s'agit (maternel ou paternel, aîné ou cadet), et je garde partout ailleurs le terme original érigé en nom propre, ou je traduis par " oncle " ou " tante ", selon les nécessités du contexte. Je ne traite pas systématiquement tous les textes de la même manière.

Dans "Concours d'agapes ", où il est question d'un mariage arrangé et de la concurrence féroce que se livrent les deux familles (celle de la fille et celle du garçon) pour régaler les centaines d'invités avec le meilleur festin dans les jours précédant la cérémonie, le père de la fille dénigre le repas à l'hôtel organisé par la famille du garçon. " No buffetshuffet for me ", proclame-t-il. Les Indiens utilisent souvent ce genre de rime, qu'ils inventent eux-mêmes, quand ils parlent en anglais : une habitude empruntée à la langue hindi où le deuxième mot sert à renforcer le premier (" shaadi vaadi ", par exemple, où seul le mot "shaadi " à un sens - mariage). L'anglais britannique possède des tournures semblables, comme " arty-farty " (prétentieux) ou " namby-pamby " (gnangnan). Cette tradition existe-t-elle en français ?

Non, mais il est très amusant de l'adopter en donnant un sens à l'association de deux éléments modifiés pour se ressembler phonétiquement, seul moyen de faire accepter ce couple étrange venu d'ailleurs sans qu'on glisse dans le calque passif. Dans la nouvelle que vous évoquez, je fais dire au père en français (en rapport avec ce qui suit) : " pas question de buffetpiaffet pour moi !... II n'y a que les chevaux pour manger debout. "

(4) Crêpe à base de riz et de lentilles.

(5) Gâteau de riz cuit à la vapeur.

(6) Plat unique à base de riz basmati préparé avec des épices et, au choix, de la viande, des œufs ou des légumes. 
Quelle recette du livre vous a vraiment mis l'eau à la bouche?

Celle du gâteau de carotte, page 48(7).

Dans un entretien avec le blog Littexpress(8) en 2013, vous avez dit ceci : " Je ne "suis" pas traductrice. Premièrement, je suis, et deuxièmement, je traduis. Je ne suis pas identifiable à mon métier. "Or le travail que nous faisons est une partie fondamentale de notre identité à nous tous : quand on rencontre un inconnu, c'est même une des premières questions qu'on lui pose...

Je sais, c'est précisément la raison pour laquelle j'ai jugé nécessaire de donner cette précision : je tiens à me dissocier de cet automatisme social. Je ne peux pas développer sans passer complètement hors sujet. Je dirai simplement que le travail, nos spécialités, nos préoccupations, nos fiertés nous font oublier des questions essentielles et contribuent à repousser les vraies priorités au second plan : notamment la nécessité de vivre tous radars sensoriels et critiques déployés et ce qu'il y a à faire afin que la Terre redevienne un endroit vivable pour tous les êtres vivants.

De tous les romans indiens que vous avez traduits (la bibliographie est longue !), quels sont vos trois coups de cœur? S'agit-il également des livres que vous avez pris le plus de plaisir à traduire?

Oui, le plaisir de traduire correspond exactement aux livres que je préfère. Cependant, je ne peux pas en choisir trois, ce serait purement arbitraire ! Jours d'amour et d'épreuve, l'histoire du roi Nala vient en tout premier, puis, ex-æquo, les romans et recueils de nouvelles traduits du malayalam, Les Légendes de Khasak de O.V. Vijayan aux éditions Fayard et les trois Basheer publiés chez Zulma : Grand-Père avait un éléphant, Les Murs et autres histoires (d'amour), Le Talisman (grand prix de traduction de la ville d'Arles en 2012). De l'anglais, j'ai aimé profondément Chamans, mystiques et médecins, de Sudhir Kakar (Seuil), Retour sur image de Mukul Kesavan (Picquier), La Découverte de l'Inde de Jawaharlal Nehru (Picquier), traduit en collaboration avec Catherine Richard, Le dernier rire du moteur d'avion, de Ruchir Joshi et le Trotter Nama d'Allan Sealy (Fayard), Cette Nuit-là, d'Indra Sinha (Albin Michel), sans oublier Manil Suri... Vous voyez, je n'arrive pas à me limiter.

chakori.dv@orange.fr

(7) Cette recette est reproduite en fin d'article avec l'aimable autorisation des Éditions Philippe Picquier.

(8) http://littexpress.over-blog.net/article-entretien-avec-dominique-vitalyos-traductrice-114299662.html 


\section{Gâteau de carotte}

$400 \mathrm{~g}$ de farine

$350 \mathrm{~g}$ de sucre en poudre blanc (de canne, de préférence)

$1 \frac{1}{2}$ tasse d'huile d'arachide

4 œufs

2 cuillères à café de poudre de cannelle

2 cuillères à café de bicarbonate de soude

1 cuillère à café de sel

$31 / 2$ tasses de carottes râpées

$200 \mathrm{~g}$ de noix broyées

$100 \mathrm{~g}$ de raisins secs et/ou de dattes

Déposez tous les ingrédients ensemble dans un grand bol et mélangez avec une spatule en bois bien solide. La pâte obtenue est collante et difficile à remuer, mais ne vous y arrêtez pas. Faites cuire à four chaud dans un grand moule huilé et fariné, entre 45 et 60 minutes. Voir se transformer cette mélasse en gâteau appétissant, fleurant bon la cannelle et d'un joli ton orangé, est toujours une surprise. Vous pouvez glacer le dessus en suivant la recette ci-dessous. Si vous savez résister, mettez-le au réfrigérateur et attendez deux jours avant de le manger, pour permettre aux saveurs de s'exalter.

\section{Glaçage au fromage blanc}

$25 \mathrm{~g}$ de sucre glace

$50 \mathrm{~g}$ de beurre fondu

$1 / 2$ cuillère à café d'essence de vanille

$200 \mathrm{~g}$ de fromage blanc

Battez le fromage blanc avec les autres ingrédients et recouvrez le gâteau de ce mélange. Si vous préférez, vous pouvez l'utiliser à d'autres fins : ajoutez-y des morceaux de mangue, des tranches d'orange ou tout autre fruit de votre choix, et servez froid en dessert. 